04

\title{
Управляемая поляризацией инверсия топологического заряда оптических вихрей в мульти-эллиптических оптических волокнах
}

\author{
() К.Н. Алексеев, Е.В. Баршак, Д.В. Викулин, Б.П. Лапин, М.А. Яворский \\ Крымский федеральный университет имени В.И. Вернадского, Физико-технический институт, \\ 295007 Симферополь, Республика Крым, Россия \\ e-mail: phystechs@cfuv.ru
}

Поступила в редакцию 27.10.2017 г.

В окончательной редакции 18.12.2017 г.

\begin{abstract}
Получены аналитические выражения для мод высших порядков с азимутальным числом $|\ell| \geq 2$ и их постоянных распространения мульти-эллиптических оптических волокон с крутильными механическими напряжениями вблизи резонансных значений шага скрутки. Показано, что резонансные моды представляют собой суперпозицию двух оптических вихрей с одинаковой циркулярной поляризацией и противоположным знаком топологического заряда. Предсказан эффект инверсии топологического заряда выходного оптического вихря, управляемой непосредственно изменением знака циркулярной поляризации входного пучка. Это открывает возможность создания логического элемента CNOT на основе мульти-эллиптических волокон.
\end{abstract}

DOI: $10.21883 /$ OS.2018.04.45755.253-17

Оптические вихри $(\mathrm{OB})$ представляют собой винтовые дислокации волнового фронта с неопределенностью фазы и, как следствие, нулевым значением интенсивности на оси пучка. Благодаря этим свойствам ОВ имеют важное прикладное значение в создании оптических пинцетов, в микроскопии и астрофизики и т.д., что подтверждается экспериментально [1-3]. В настоящее время ОВ приобретают все большее значение в информационных технологиях, что связано с их способностью переносить орбитальный угловой момент (ОУМ). Действительно, форма волнового фронта ОВ в виде геликоида с $\ell$-ветвями, где $\ell-$ его топологический заряд (ТЗ), приводит к появлению поперечной компоненты вектора Пойнтинга и, как следствие, возникновению ОУМ, в значениях которого кодируется информация. ОУМ на один фотон определяется выражением $L=\hbar \ell$, где $\hbar-$ приведенная постоянная Планка. Так как $\ell=0, \pm 1, \pm 2, \ldots$, набор возможных значений ОУМ теоретически не ограничен, что позволяет кодировать несоизмеримо больше информации в сравнении со стандартными техниками. Таким образом, ОУМ-мультиплексирование существенно увеличивает пропускную способность канала связи и, кроме того, позволяет достичь принципиально нового уровня защиты данных [4].

Естественно, для полноценной реализации ОУМмультиплексирования необходим способ передачи ОВ без искажений на необходимые расстояния. С этой целью были исследованы процессы распространения ОВ как в свободном пространстве [5-7], так и в оптических волокнах различного типа [8-13]. Было показано, однако, что основанная на ОУМ передача информации в свободном пространстве испытывает серьезные ограничения, вызванные разрушающим влиянием атмосферы [14-16]. Таким образом, более перспективной средой для передачи информации в значениях ОУМ ОВ оказались оптические волокна. Хотя устойчивость ОВ во мно- гих исследованных типах волокон носит существенно ограниченный характер $[17,18]$, следует отметить, что была продемонстрирована принципиальная возможность использования волокон с крутильными механическими напряжениями (КМН) для устойчивого по отношению к малым внешним возмущениям распространения ОВ, в частности для моделей с анизотропией материала и эллиптической формой поперечного сечения [19,20].

Помимо вопросов, связанных с передачей данных и генерацией пучков с ОУМ, успешное применение ОВ в информационных технологиях требует также решения других задач, одной из которых является проблема реализации базовых логических операций над значениями ОУМ. Так как ортогональные состояния ОВ с различными значениями ОУМ образуют многомерное пространство, этот вопрос оказывается тесно связанным с возможностью моделирования квантовых вычислений при помощи классических оптических полей [21-23]. Так, фундаментальная способность квантовых систем образовывать перепутанные состояния успешно моделируется при помощи специфических манипуляций с оптическими пучками [24], в частности с использованием оптических волокон [25-28]. Однако в настоящее время для этих целей используются только волоконные моды высших порядков, имеющие нулевое значение ОУМ, такие как TE-, TM- [26], HG- [27] и LP-моды [28]. Очевидно, что для полноценного использования информационного потенциала состояний фотонов с ОУМ в области моделирования квантовых вычислений и коммуникаций требуется механизм генерации перепутанных состояний $\mathrm{OB}$, а также осуществления базовых логических операций - гейтов. Таким образом, целью настоящей работы является: (i) установление вида мод высших порядков с азимутальным числом $|\ell| \geq 2$ мульти-эллиптических волокон (МЭВ) с КМН вблизи резонансных значений шага скрутки и (ii) демонстрация способности данных 
волокон осуществлять управляемую поляризацией инверсию ТЗ ОВ. Данный эффект позволяет реализовать полностью волоконный фундаментальный логический элемент CNOT, являющийся неотъемлемой частью всех основных квантовых алгоритмов и позволяющий генерировать перепутанные состояния для ОВ с ТЗ $|\ell|>1$.

\section{Модель и основное уравнение}

Модель МЭВ с КМН описывается следующим тензором диэлектрической проницаемости (рис. 1):

$$
\hat{\varepsilon}_{\mathrm{MHF}}(r, \varphi)=\varepsilon_{\mathrm{IF}}(r) \hat{1}+\varepsilon_{\mathrm{Mh}}(r, \varphi) \hat{1}+\hat{\varepsilon}_{\mathrm{TMS}}(r, \varphi) .
$$

Здесь слагаемое $\varepsilon_{\mathrm{IF}}(r)=\varepsilon_{\mathrm{co}}[1-2 \Delta f(r)]$ описывает диэлектрическую проницаемость идеального циркулярного волокна (ЦВ), $\Delta=\left(\varepsilon_{\mathrm{co}}-\varepsilon_{\mathrm{cl}}\right) / 2 \varepsilon_{\mathrm{co}}-$ высота профиля диэлектрической проницаемости, $\varepsilon_{\mathrm{co}}$ и $\varepsilon_{\mathrm{cl}}-$ значения диэлектрической проницаемости в сердцевине и оболочке волокна соответственно. Для рассматриваемых в настоящей работе слабонаправляющих оптических волокон $\Delta \ll 1$, что обусловливает параксиальный характер распространения оптических мод. Функция $f(r)$ определяет распределение показателя преломления среды и для изучаемых здесь ступенчатых волокон имеет вид

$$
f(r)= \begin{cases}0, & r \leq r_{0} \\ 1, & r>r_{0},\end{cases}
$$

где $r_{0}$ - радиус сердцевины, а радиус оболочки предполагается бесконечным [29]. Используется цилиндрическая система координат $(r, \varphi, z)$, ось $z$ которой совпадает с осью волокна.

Второе слагаемое в (1) характеризует мультиэллиптическую форму поперечного сечения (анизотропия формы) [30]:

$$
\varepsilon_{\mathrm{Mh}}(r, \varphi)=-\delta \varepsilon_{\mathrm{Mh}} r f_{r}^{\prime} \cos \tau \varphi .
$$

В формуле (2) $\delta \varepsilon_{\text {Mh }}=2 \delta \Delta \varepsilon_{\text {co }}$, где параметр $\delta \ll 1$ характеризует степень деформации поперечного сечения, а параметр $\tau=2,4,6, \ldots$ определяет порядок симметрии поперечного сечения волокна. Заметим, что в простейшем случае эллиптической формы сечения волокна $(\tau=2)$ параметр $\delta$ связан с эксцентриситетом эллипса, $e=\sqrt{1-(a / b)^{2}}$, где $a, b-$ полуоси, выражением $e=2 \sqrt{\delta} /(1+\delta)$, при малых $\delta$ переходящим в $e \approx 2 \sqrt{\delta}$.

Последнее слагаемое в (1) описывает влияние KMH [20],

$$
\hat{\varepsilon}_{\mathrm{TMS}}(r, \varphi)=\delta \varepsilon_{\mathrm{TMS}} R\left(\begin{array}{ccc}
0 & 0 & \sin \varphi \\
0 & 0 & -\cos \varphi \\
\sin \varphi & -\cos \varphi & 0
\end{array}\right)
$$

где $\delta \varepsilon_{\mathrm{TMS}}=q p_{44} \varepsilon_{\mathrm{co}}^{2} r_{0}, q=2 \pi / H, H-$ шаг скрутки, константа фотоупругости $p_{44}=-0.075$ (для кварца),
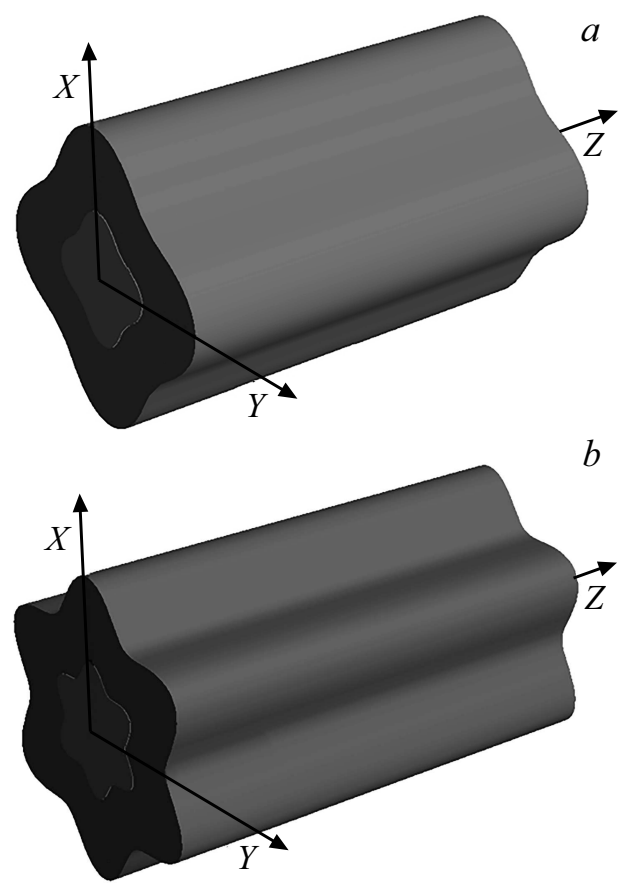

Рис. 1. Модели МЭВ волокон при различных значениях параметра $\tau$, определяющего порядок симметрии поперечного сечения волокна: $(a) \tau=4$, (b) $\tau=6$.

$R=r / r_{0}$. Отметим, что $\delta \varepsilon_{\mathrm{Mh}}, \delta \varepsilon_{\mathrm{TMS}} \ll \varepsilon_{\mathrm{IF}}(r)$, т. е. КМН и анизотропия формы представляют собой малые поправки к скалярной диэлектрической проницаемости.

Распространение света в оптическом волокне описывается векторным волновым уравнением

$$
\begin{aligned}
& \left(\nabla^{2}+k^{2} \hat{\varepsilon}(x, y, z)\right) \mathbf{E}(x, y, z) \\
& \quad=-\nabla\left[\left(\mathbf{E} \nabla \ln \varepsilon_{\mathrm{IF}}\right)+q p_{44} \varepsilon_{\mathrm{co}} r\left(\sin \varphi \frac{\partial E}{\partial z}+\cos \frac{\partial E_{y}}{\partial z}\right)\right],
\end{aligned}
$$

где $k=2 \pi / \lambda-$ волновой вектор в вакууме, $\lambda-$ длина волны, $\mathbf{E}$ - напряженность электрического поля. Уравнение (4) удобно записать в операторной форме

$$
\left(\hat{H}_{\mathrm{IF}}+\hat{V}_{\mathrm{TMS}}+\hat{V}\right)|\Psi\rangle=\beta|\Psi\rangle .
$$

Здесь $\hat{H}_{\mathrm{IF}}$ имеет стандартный вид [29] и управляет распространением света в ЦВ с учетом спин-орбитального взаимодействия (COB), оператор $\hat{V}_{\mathrm{TMS}}=k^{2} \hat{\varepsilon}_{\mathrm{TMS}}(r, \varphi)$ обусловлен влиянием КМН, а оператор $\hat{V}$ в случае МЭВ имеет вид $\hat{V}=k^{2} \varepsilon_{\mathrm{Mh}}(r, \varphi) \hat{1},|\Psi\rangle=\operatorname{col}\left(E_{x}, E_{y}, E_{z}\right)$. Отметим, что в уравнении (5) имеет место влияние поперечной $E_{t}$ и продольной $E_{z}$ компонент электрического поля на пространственную эволюцию друг друга, что выражается в невозможности записать замкнутую систему уравнений относительно $E_{t}$. Однако относительная малость продольной компоненты в сравнении с поперечной для исследуемых слабонаправляющих волокон $(\Delta \ll 1, r \gg \lambda)$ позволяет пренебрегать таким 
влиянием, так что обычно работают только с поперечной составляющей вектора электрического поля $E_{t}$ [29]. Между тем, как следует из вида показателя преломления (1) и поправки (2), для учета влияния КМН на распространение света необходимо учитывать продольную компоненту $E_{z}$, несмотря на ее относительную малость. Так как для всех практически значимых параметров волокна имеет место соотношение $\hat{H}_{\mathrm{IF}} \gg \hat{V}_{\mathrm{TMS}}, \hat{V}$, то для решения уравнения (5) можно применять теорию возмущений.

\section{Структура мод и постоянные распространения}

Согласно методу теории возмущений, моды и их постоянные распространения являются решениями уравнения вида

$$
\hat{H} x_{k}=0 \text {, }
$$

где матрица возмущений $\hat{H}$ строится стандартным способом путем усреднения векторного волнового уравнения на множестве собственных функций невозмущенного волокна $\left|\Phi_{i}\right\rangle$, компоненты векторов $\mathbf{x}_{k}$ суть коэффициенты $a_{k}^{i}$ в разложении искомых мод $|\Phi\rangle_{k}$ по модам невозмущенного волокна: $|\Psi\rangle_{k}=\sum_{i} a_{k}^{i}\left|\Phi_{i}\right\rangle$. Далее нас будут интересовать волокна, поддерживающие распространение мод с азимутальным числом $|\ell| \geq 2$. Строго говоря, базис теории возмущений при этом должен состоять из всех мод невозмущенного волокна. Возмущение волокна может привести к гибридизации мод невозмущенного волокна как с одинаковыми значениями азимутального числа, так и с разными. Однако благодаря существенному различию скалярных постоянных распространения для мод с разными азимутальными числами [29] имеет место широкий диапазон параметров волокна, в котором можно пренебречь связью таких мод, индуцированной рассматриваемыми возмущениями. Поэтому в настоящей работе мы ограничимся исследованием в пределах групп мод с одинаковым азимутальным числом. Отметим также, что при исследовании направляемых мод слабонаправляющих оптических волокон в волоконной оптике обычно не учитывают незначительную долю энергии, приходящуюся на оболочечные моды [29]. Связью этих мод с сердцевинными модами волокна можно пренебречь, принимая во внимание разницу между их скалярными постоянными распространения, имеющими соответственно порядок $\propto \sqrt{\varepsilon_{\mathrm{cl}}} \beta_{m}$ и $\propto \sqrt{\varepsilon_{\mathrm{co}}} \beta_{m}$, так что возмущения волокна не могут привести к существенной связи этих полей и изменению структуры исследуемых в настоящей работе направляемых мод.

Базисные векторы теории возмущений $\left|\Phi_{i}\right\rangle$ являются собственными функциями оператора нулевого приближения $\hat{H}_{\mathrm{IF}}$ и, как известно, представляют собой циркулярно-поляризованные ОВ [8]:

$$
|1, m\rangle,|1,-m\rangle,|-1,-m\rangle,|-1, m\rangle
$$

где

$$
\begin{aligned}
|\sigma, \ell\rangle= & \frac{1}{\sqrt{2}} e^{i \ell \varphi} \\
& \times \operatorname{col}\left(F_{m}(r), i \sigma F_{m}(r), \frac{i}{\tilde{\beta}_{m} r}\left[r F_{m}^{\prime}-\sigma \ell F_{m}(r) e^{i \sigma \varphi}\right]\right),
\end{aligned}
$$

индекс определяет направление циркулярной поляризации, $m=|\ell|$. Радиальная функция $F_{m}(R)$ является решением известного уравнения

$$
\left(\frac{\partial^{2}}{\partial R^{2}}+\frac{1}{R} \frac{\partial}{\partial R}-\frac{l^{2}}{R^{2}}+\tilde{U}^{2}-V^{2} f(R)\right) F_{m}(R)=0,
$$

где $V=k r_{0}\left(\varepsilon_{\mathrm{co}}-\varepsilon_{\mathrm{cl}}\right)^{1 / 2}=k r_{0} \sqrt{2 \Delta \varepsilon_{\mathrm{co}}}-$ волноводный параметр, $\tilde{U}=r_{0}\left(k^{2} \varepsilon_{\text {со }}-\tilde{\beta}^{2}\right)^{1 / 2}, \tilde{\beta}_{m}-$ скалярная постоянная распространения. Для волокон со ступенчатым профилем показателя преломления $F_{m}(R)$ имеет вид

$$
F_{m}(R)=\left\{\begin{array}{ll}
J_{m}(\tilde{U} R) / J_{m}(\tilde{U}), & r \leq r_{0} \\
K_{m}(\tilde{W} R) / K_{m}(\tilde{W}), & r \geq r_{0}
\end{array},\right.
$$

где $J_{m}-$ функция Бесселя 1-го рода $m$-го порядка, а $K_{m}$ - модифицированная функция Бесселя 1-го рода $m$-го порядка (модифицированная функция Ханкеля), $\tilde{W}^{2}=V^{2}-\tilde{U}^{2}$

Усреднение полного оператора возмущенного волокна в уравнении (5) на состояниях (7) позволяет получить следующую матрицу возмущений для МЭВ:

$$
\hat{H}_{m}=\left(\begin{array}{cccc}
P_{m} & D_{\tau, m} & 0 & 0 \\
D_{\tau, m} & L_{-m} & 0 & 0 \\
0 & 0 & P_{-m} & D_{\tau, m} \\
0 & 0 & D_{\tau, m} & L_{m}
\end{array}\right)
$$

где $P_{ \pm m}=-\beta^{2}+\tilde{\beta}_{m}^{2}+A_{m}+\gamma_{ \pm(m+1)}, L_{ \pm m}=-\beta^{2}+\tilde{\beta}_{m}^{2}+$ $+B_{m}+\gamma_{ \pm(m-1)}$. Матричные элементы $\gamma_{M}=-2 \tilde{\beta}_{m} M g q$ описывают влияние КМН на структуру искомых мод, $g=\frac{1}{2} \varepsilon_{\mathrm{co}}\left|p_{44}\right|, M=\sigma+\ell-$ полный угловой момент. Элементы $D_{\tau, m}=-\delta_{\tau, 2 m} k^{2} \delta \varepsilon_{\mathrm{Mh}} / 2 Q_{m}$, где $\delta_{\tau, 2 m}-$ символ Кронекера, характеризуют влияние анизотропии формы поперечного сечения. Таким образом, в использованном приближении для модели волокна с заданной мультиэллиптичностью поперечного сечения $\tau$ эффект межмодовой связи имеет место только для группы невозмущенных мод с азимутальным числом $m=\tau / 2$. Константы $A_{m}=\frac{\Delta}{r_{0}^{2} Q_{m}}\left(F_{m}^{\prime}(1)-m\right)$ и $B_{m}=-\frac{\Delta}{r_{0}^{2} Q_{m}}\left(F_{m}^{\prime}(1)+m\right)$ характеризуют СОВ, $Q_{m}=\int_{0}^{\infty} R F_{m}^{2}(R) d R$.

Решение уравнения (6) с матрицей возмущения (8) позволяет получить выражения для искомых мод и их 


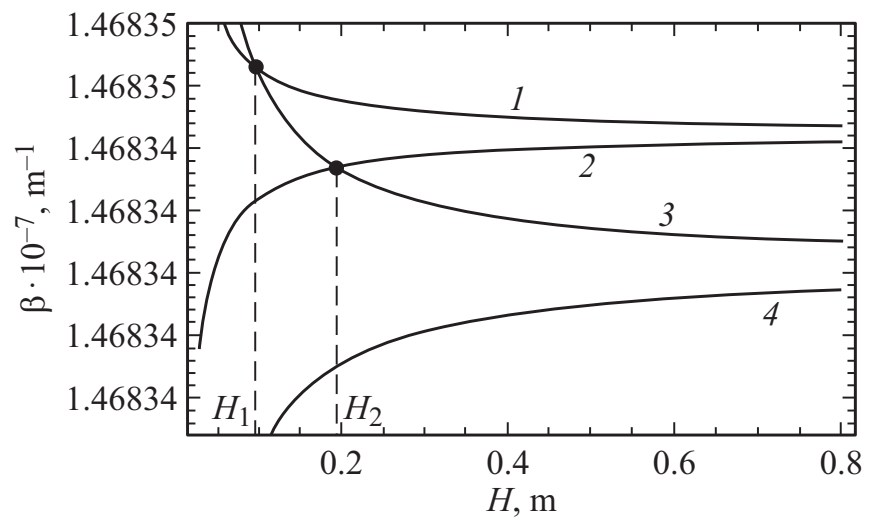

Рис. 2. Постоянные распространения мод невозмущенного волокна (9). Кривая 1 соответствует постоянной распространения поля $|1,-m\rangle, 2-$ поля $|-1, m\rangle, 3-|-1,-m\rangle$, $4-|1, m\rangle$. При $H=H_{1}$ имеет место случайное вырождение уровней $\mathrm{OB}|-1,-m\rangle$ и $|1,-m\rangle$, а при $H=H_{2}-$ полей $|-1,-m\rangle$ и $|-1, m\rangle$. Параметры волокна: $V=7.06$, $r_{0}=12 \lambda_{\mathrm{He}-\mathrm{He}}, p_{44}=0.075, \delta=10^{-4}, \tau=4$.

постоянных распространения при произвольных разумных параметрах волокна. Далее, однако, мы остановимся на рассмотрении важного с практической точки зрения частного случая, когда влияние анизотропии формы мало по сравнению с СОВ и КМН: $\delta \varepsilon_{\text {Mh }} \ll q / k$, $\Delta /\left(k r_{0}\right)^{2}$. Процедура получения мод в таком режиме выглядит физически более содержательно, если использовать теорию возмущений, определив естественным образом в качестве оператора нулевого приближения оператор $\hat{H}_{m}\left(D_{\tau, m}=0\right)$, что установит оператор $\hat{V}_{\tau, m}=\hat{H}_{\tau, m}-\hat{H}_{m}\left(D_{\tau, m}=0\right)$ в качестве возмущения. Решение уравнения вида (6) для оператора $\hat{H}_{m}\left(D_{\tau, m}=0\right)$ дает следующий результат: его собственные векторы представляют собой 4 циркулярно-поляризованных OB (7), а соответствующие им постоянные распространения имеют вид (рис. 2)

$$
\begin{aligned}
& \beta_{ \pm 1, \pm m}=\tilde{\beta}_{\mathrm{HE}_{m+1}} \mp(m+1) g q, \\
& \beta_{ \pm 1, \mp m}=\tilde{\beta}_{\mathrm{HE}_{m-1}} \pm(m-1) g q,
\end{aligned}
$$

где подразумевается использование либо верхнего, либо нижнего набора знаков,

$$
\tilde{\beta}_{\mathrm{HE}_{m+1}}=\tilde{\beta}_{m}+\frac{A_{m}}{2 \tilde{\beta}_{m}}, \quad \tilde{\beta}_{\mathrm{HE}_{m-1}}=\tilde{\beta}_{m}+\frac{B_{m}}{2 \tilde{\beta}_{m}} .
$$

Важно отметить, что зависимость спектра (9) от величины шага скрутки через $q$ определяет степень влияния анизотропии на собственные состояния с $m \geq 2$ рассматриваемых волокон. Как видно из рис. 2 , существуют такие параметры шага скрутки, при которых спектральные кривые (9) хорошо разнесены. Тогда учитывать влияние анизотропии формы следует согласно теории возмущений без вырождения, что приведет лишь к незначительной перенормировке спектра, а модовая структура останется неизменной. В этом случае можно говорить о структурной устойчивости ОВ и использовании МЭВ для устойчивой передачи информации в значениях их ОУМ. Отметим, что критерий структурной устойчивости мод определяется в рамках стандартного подхода теории возмущений: моды являются устойчивыми к внешним возмущениям $\delta \hat{V}$, когда расстояние между их постоянными распространения существенно превосходит соответствующий матричный элемент, $\left|\beta_{i}^{2}-\beta_{k}^{2}\right| \gg\left|\left\langle\Psi_{i}|\delta \hat{V}| \Psi_{k}\right\rangle\right|$, т.е. малые внешние возмущения не могут обеспечить эффективную гибридизацию мод волокна.

С другой стороны, существуют такие параметры волокна, при которых спектр (9) оказывается двукратно вырожденным. Из выражений (9) легко найти условия, определяющие резонансные значения шага скрутки волокна (или, что эквивалентно, значения $q$ ):

$$
\begin{aligned}
\beta_{1,-m} & =\beta_{-1,-m} \Rightarrow q_{1} \\
& =\frac{B_{m}-A_{m}}{4 \tilde{\beta}_{m}(m+1-(m+1)) g}=\frac{B_{m}-A_{m}}{4 \tilde{\beta}_{m} g}, \\
\beta_{-1,-m} & =\beta_{-1, m} \Rightarrow q_{2} \\
& =\frac{B_{m}-A_{m}}{4 \tilde{\beta}_{m}(m+1+(m-1)) g}=\frac{B_{m}-A_{m}}{4 m \tilde{\beta}_{m} g} .
\end{aligned}
$$

Вблизи таких точек имеет место неустойчивость системы относительно даже малого возмущения.

\section{Резонансные моды}

Пусть шаг скрутки МЭВ близок к резонансному значению, когда анизотропия формы может привести к существенному изменению структуры мод путем сильной гибридизации нулевых мод невозмущенного волокна (7). Действительно, как видно из рис. 2, при $q=q_{1}\left(H=H_{1}\right)$ возможна эффективная связь между полями $|-1,-m\rangle$ и $|1,-m\rangle$, а при $q=q_{2}\left(H=H_{2}\right)-$ между полями $|-1,-m\rangle$ и $|-1, m\rangle$. Однако, как следует из матрицы (8), в исследуемом волокне нет возмущения, которое могло бы гибридизовать поля $|-1,-m\rangle$ и $|1,-m\rangle$, поэтому, несмотря на случайное вырождение спектра, структура мод волокна вблизи резонансной точки $H=H_{1}$ останется прежней. В то же время, вблизи резонансной точки $H=H_{2}$ поля с одинаковой поляризацией и противоположным знаком Т3 $|-1,-m\rangle$ и $|-1, m\rangle$ могут эффективно связываться малой анизотропией формы, поэтому далее получим структуру мод волокна вблизи этой резонансной точки.

Когда шаг скрутки близок к значению $\mathrm{H}=\mathrm{H}_{2}$, можно ограничиться рассмотрением подпространства, состоящего только из двух вырожденных мод нулевого приближения. Таким образом, вместо четырехмерной матрицы возмущений мы получаем двумерную матрицу 
для резонансной точки $q=q_{2}(10)$ :

$$
\hat{H}_{2}=\left(\begin{array}{cc}
P_{-m} & D_{\tau, m} \\
D_{\tau, m} & L_{m}
\end{array}\right),
$$

оператор $\hat{H}_{2}$ определен на состояниях $\{|-1,-m\rangle,|-1, m\rangle\}$. Решение уравнения вида (6) для оператора (11) позволяет получить структуру мод МЭВ вблизи резонансной точки $\mathrm{H}=\mathrm{H}_{2}$ :

$$
\begin{aligned}
& \left|\Psi_{1}\right\rangle=\cos \alpha|-1,-m\rangle+\sin \alpha|-1, m\rangle, \\
& \left|\Psi_{2}\right\rangle=\sin \alpha|-1,-m\rangle-\cos \alpha|-1, m\rangle,
\end{aligned}
$$

где $\cos 2 \alpha=m g \varepsilon / \Omega, \varepsilon=q-q_{2}, \quad \Omega=\sqrt{(m g \varepsilon)^{2}+T_{\tau, m}^{2}}$, $T_{\tau, m}=D_{\tau, m} / 2 \tilde{\beta}_{m}, 0 \leq \alpha \leq \frac{\pi}{4}$. Постоянные распространения мод (12) имеют вид

$$
\beta_{1,2}=\tilde{\beta}_{-1,-m}-m g \varepsilon \pm \Omega
$$

Полученные резонансные моды (12) являются гибридными: состоят из двух ОВ с одинаковым значением циркулярной поляризации и противоположными знаками Т3. Распределение энергии между парциальными полями мод (12) контролируется параметром $\alpha$, который определяется величиной анизотропии формы и близостью величины шага скрутки волокна к резонансному значению. Наиболее сильная связь между модами невозмущенного волокна (7) возникает, когда условие резонанса $q=q_{2}$ строго выполняется, при этом $\varepsilon=0$ и $\alpha=\pi / 4$. В спектре при этом в результате влияния анизотропии формы снимается вырождение вблизи резонансной точки (рис. 3). Когда реализуется предельный случай $\alpha \rightarrow 0$, т.е. область рассмотрения удаляется от резонансных точек, гибридные моды асимптотически переходят в моды невозмущенного волокна (7).

\section{Управляемая поляризацией инверсия топологического заряда}

Используя выражения (12) и (13), покажем, как МЭВ при определенных параметрах могут быть применены для контролированного изменения знака Т3 (инверсии) выходного пучка путем управления знаком циркулярной поляризации входного ОВ. Пусть левоциркулярно поляризованный ОВ с Т3 $\ell=-m$ возбуждается на входном торце МЭВ с величиной параметра скрутки $q$, близкой к резонансному значению $q_{2}:\left|\Psi_{\Pi}\right\rangle=|-1,-m\rangle$. Такой ОВ возбуждает в волокне следующую суперпозицию мод:

$$
|\Psi(z)\rangle=s_{1}\left|\Psi_{1}\right\rangle e^{i \beta_{1} z}+s_{2}\left|\Psi_{2}\right\rangle e^{i \beta_{2} z} .
$$

Граничное условие $|-1,-m\rangle=s_{1}\left|\Psi_{1}\right\rangle+s_{2}\left|\Psi_{2}\right\rangle$ позволяет найти коэффициенты разложения $s_{i}$ в (14), что

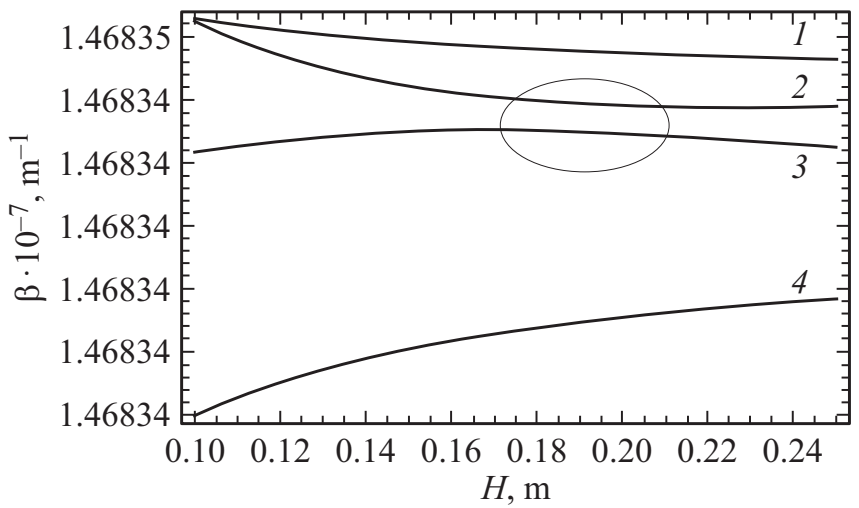

Рис. 3. Снятие вырождения спектра вблизи резонансной точки за счет действия анизотропии формы. Кривая 1 соответствует постоянной распространения поля $|1,-m\rangle$, кривые 2 и 3 постоянным распространения гибридных мод $\left|\Psi_{1}\right\rangle$ и $\left|\Psi_{2}\right\rangle$ (см. (13)), кривая 4 - постоянной распространения ОВ $|1, m\rangle$. Параметры волокна: $V=7.06, r_{0}=12 \lambda_{\mathrm{He}-\mathrm{He}}, p_{44}=0.075$, $\delta=10^{-4}, \delta=10^{-4}, \tau=4$.

приводит к следующему выражению для поля $|\Psi(z)\rangle$ :

$$
|\Psi(z)\rangle=b_{1}(z)|-1,-m\rangle+b_{2}(z)|-1, m\rangle .
$$

Коэффициенты $b_{i}(z)$ записываются в виде

$$
\begin{gathered}
b_{1}(z)=\cos (\Omega z)+i \frac{m g \varepsilon}{\Omega} \sin (\Omega z), \\
b_{2}(z)=i \frac{T_{\tau, m}}{\Omega} \sin (\Omega z) .
\end{gathered}
$$

Отметим, что для коэффициентов (16) справедливо $\left|b_{1}\right|^{2}+\left|b_{2}\right|^{2}=1$. Из выражения (15) видно, что поле в волокне состоит из падающего ОВ $|-1,-m\rangle$ и генерируемого ОВ $|-1, m\rangle$ с энергией $W_{-1, m}=\left|b_{2}\right|^{2}$ :

$$
\begin{aligned}
W_{-1, m}(\varepsilon, z)= & \frac{D_{\tau, m}^{2}}{\left(2 \tilde{\beta}_{m} m g \varepsilon\right)^{2}+D_{\tau, m}^{2}} \\
& \times \sin ^{2}\left(z \sqrt{(m g \varepsilon)^{2}+\left(D_{\tau, m} / 2 \tilde{\beta}_{m}\right)^{2}}\right) .
\end{aligned}
$$

Из (17) следует, что на расстоянии $z=L_{n}$, где

$$
L_{n}=\frac{\pi}{2 \sqrt{(m g \varepsilon)^{2}+\left(D_{\tau, m} / 2 \tilde{\beta}_{m}\right)}}(2 n+1),
$$

$n=0,1,2, \ldots$, доля энергии, сконцентрированная в парциальном вихре $|-1, m\rangle$, достигает своего максимального значения

$$
W_{-1, m}\left(\varepsilon, L_{n}\right)=\frac{D_{\tau, m}^{2}}{\left(2 \tilde{\beta}_{m} m g \varepsilon\right)^{2}+D_{\tau, m}^{2}} .
$$

Легко видеть, что при выполнении резонансного условия $\varepsilon=0, W_{-1, m}\left(0, L_{n}\right)=1$ и, следовательно, вся падающая энергия переходит в левоциркулярно поляризован- 


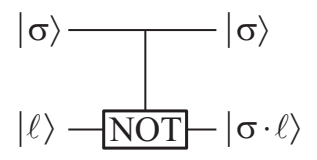

Рис. 4. Схема логического элемента „Управляемое-НЕ“ (CNOT gate): роль управляющего кубита играет состояние циркулярной поляризации $\sigma= \pm 1$, а управляемого - Т3 ОВ $\ell= \pm m$.

ный $\mathrm{OB}|-1, m\rangle$, т.е. имеет место процесс инверсии Т3 со 100\%-ной эффективностью. Легко понять, что в случае падающего ОВ вида $|-1 . m\rangle$ получим конверсию $|-1, m\rangle \rightarrow|-1,-m\rangle$. Вспоминая, что ТЗ связан с азимутальным числом как $\ell= \pm m$, можно объединить два этих случая:

$$
|-1, \ell\rangle \rightarrow|-1,-\ell\rangle .
$$

Необходимо подчеркнуть, что вблизи данного резонанса в силу введного выше условия малости анизотропии формы ОВ $|1, m\rangle$ и $|1,-m\rangle$ с высокой степенью точности являются модами, и поэтому не меняют своей структуры при распространении:

$$
|1, \ell\rangle \rightarrow|1, \ell\rangle
$$

Таким образом, выражения (18) и (19) указывают на возможность управления знаком Т3 выходного ОВ с помощью МЭВ с КМН путем управления знаком циркулярной поляризации входного ОВ, что демонстрирует теоретическую возможность создания фундаментального логического элемента — „Управляемое-НЕ“ (CNOT gate), где роль управляющего кубита играет состояние циркулярной поляризации $\sigma= \pm 1$, а управляемого - Т3 ОВ $\ell= \pm m$ (рис. 4). Стоит отметить, что гибридные моды (12) не чувствительны к малым отклонениям параметров волокна, что позволяет говорить о достаточной устойчивости процесса преобразования (18).

В качестве конкрентного примера приведем МЭВ с волноводным параметром $V=7.06$, радиусом сердцевины $r_{0}=12 \lambda_{\mathrm{He}-\mathrm{He}}$, параметром симметрии поперечного сечения $\tau=4, \delta=10^{-4}$. Тогда резонансное значение шага скрутки $H_{2} \approx 0.19 \mathrm{~m}$, а длина конверсии Т3 ОВ с $m=2$ составляет $L_{0} \approx 1.48 \mathrm{~m}$. Как следует из выражения (17), с увеличением анизотропии формы $\delta$ рабочая длина волокна уменышается.

В заключение необходимо отметить, что предложенный волоконный метод реализации гейта CNOT имеет важные преимущества в виде „чистоты“ волоконных полей, эффективности и устойчивости, способствуя дальнейшему расширению информационных возможностей, основанных на ОУМ-технологиях.

Исследование выполнено при финансовой поддержке Российского фонда фундаментальных исследований в рамках научного проекта № 16-47-910585.

\section{Список литературы}

[1] Grier D.G. // Nature. 2003. V. 424. P. 810-816.

[2] Spektor B., Normatov A., Shamir J. // Appl. Opt. 2008. V. 47. P. A78-A87.

[3] Foo G., Palacios D.M., Shwartzlander G.A. // Opt. Lett. 2005. V. 30. P. 3308-3310.

[4] Franke-Arnold S., Barnett S., Yao E., Leach J., Courtial J., Padgett M. // New J. Phys. 2004. V. 6. P. 103.

[5] Gibson G., Courtial J., Padgett M., Vasnetsov M., Pas'ko V., Barnett S., Franke-Arnold S. // Opt. Express. 2004. V. 12. P. 5448-5456.

[6] Bouchal Z., Chelechovsky R. // New J. Phys. 2004. V. 6. P. 131-145.

[7] Chelechovsky R., Bouchal Z. // New J. Phys. 2007. V. 9. P. 328.

[8] Alexeyev C.N., Volyar A.V., Yavorsky M.A. // Lasers, Optics and Electro-Optics Research Trends. / Ed. by Chen L.I. NY: Nova Publishers, 2007. P. 131-223.

[9] Bozinovic N., Yue Y., Ren Y., Tur M., Kristensen P., Huang H., Willner A.E., Ramachandran S. // Science. 2013. V. 340. P. $1545-1548$.

[10] Alexeyev C.N., Volyar A.V., Yavorsky M.A. // J. Opt. A: Pure Appl. Opt. 2004. V. 6. P. 162.

[11] Alexeyev C.N., Yavorsky M.A. // J. Opt. A: Pure Appl. Opt. 2004. V. 6. P. 824.

[12] Barlow A.J., Ramskov-Hansen J.J., Payne D.N. // Appl. Opt. 1981. V. 20. P. 2962-2968.

[13] McGloin D., Simpson N.B., Padgett M.J. // Appl. Opt. 1998. V. 37. P. 469.

[14] Paterson C. // Phys. Rev. Lett. 2005. V. 94. P. 153901.

[15] Tyler G., Boyd R.W. // Opt. Lett. 2009. V. 34. P. 142-144.

[16] Gbur G., Tyson R.K. // JOSA A. 2008. V. 25. P. 225-230.

[17] Alexeyev C.N., Volyar A.V., Yavorsky M.A. // J. Opt. A: Pure Appl. Opt. 2007. V. 9. P. 387-395.

[18] Alexeyev C.N. // J. Opt. 2012. V. 14. P. 085702.

[19] Barshak E.V., Alexeyev C.N., Lapin B.P., Yavorsky M.A. // Phys. Rev. A. 2015. V. 91. N 3. P. 033833.

[20] Alexeyev C., Barshak E., Fridman Y., Yavorsky M. // Appl. Opt. 2012. V. 51. N 10. P. 163-169.

[21] Cerf N.J., Adami C., Kwiat P.G. // Phys. Rev. A. 1998. V. 57. N 3. P. R1477.

[22] Spreeuw R.J.C. // Phys. Rev. A. 2001. V. 63. N 6. P. 062302.

[23] Spreeuw R.J.C. // Found. Phys. 1998. V. 28. N 3. P. 361.

[24] Lee K.F., Thomas J.E. // Phys. Rev. Lett. 2002. V. 88. N 9. P. 097902.

[25] Fu J. // Proc. SPIE. 2003. V. 5105. P. 225.

[26] Fu J., Si Z., Tang S., Deng J. // Phys. Rev. A. 2004. V. 70. N 4. P. 042313.

[27] Loffler W., Euser T.G., Eliel E.R., Scharrer M., Russel P.S.J., Woerdman J.P. // Phys. Rev. Lett. 2011. V. 106. N 24. P. 240505.

[28] Kang Y., Ko J., Lee S.M., Choi S.-K., Kim B.Y., Park H.S. // Phys. Rev. Lett. 2012. V. 109. N 24. P. 020502.

[29] Snyder W., Love J.D. Optical Waveguide Theory. London: Chapman and Hall, 1985.

[30] Alexeyev C.N., Alexeyev A.N., Lapin B.P., Milione G., Yavorsky M.A. // Phys. Rev. A. 2013. V. 88. P. 063814. 